\title{
BMJ Open Cerebrospinal fluid leakage costs after craniotomy and health economic assessment of incidence reduction from a hospital perspective in the Netherlands
}

\author{
Chris van Lieshout, ${ }^{1}$ Emma M H Slot (10 , ${ }^{2,3}$ Ahmet Kinaci, ${ }^{2}$ Mare H Kollen, ${ }^{2}$ \\ Eelco W Hoving, ${ }^{2,4}$ Geert W J Frederix, ${ }^{1}$ Tristan P C van Doormaal ${ }^{2,5}$
}

To cite: van Lieshout C, Slot EMH, Kinaci A, et al. Cerebrospinal fluid leakage costs after craniotomy and health economic assessment of incidence reduction from a hospital perspective in the Netherlands. BMJ Open 2021;11:e052553. doi:10.1136/ bmjopen-2021-052553

- Prepublication history and additional supplemental material for this paper are available online. To view these files, please visit the journal online (http://dx.doi.org/10.1136/ bmjopen-2021-052553).

CVL and EMHS contributed equally.

Received 21 April 2021 Accepted 25 November 2021

Check for updates

(c) Author(s) (or their employer(s)) 2021. Re-use permitted under CC BY-NC. No commercial re-use. See rights and permissions. Published by BMJ.

For numbered affiliations see end of article.

Correspondence to

Emma M H Slot;

e.m.h.slot-4@umcutrecht.nl

\section{ABSTRACT}

Objectives We aim to quantify the cost difference between patients with incisional cerebrospinal fluid (iCSF) leakage and those without after intradural cranial surgery. Second, the potential cost savings per patient when a decrease in iCSF leakage rate would be achieved with and without added costs for preventative measures of various price and efficacy are modelled.

Design Health economic assessment from a hospital perspective based on a retrospective cohort study. Setting Dutch tertiary referral centre.

Participants We included 616 consecutive patients who underwent intradural cranial surgery between 1 September 2017 and 1 September 2018. Patients undergoing burr hole surgery or transsphenoidal surgery, or who died within 1 month after surgery or were lost to follow-up were excluded.

Primary and secondary outcome measures Outcomes of the cost analysis include a detailed breakdown of mean costs per patient for patients with postoperative iCSF leakage and patients without, and the mean cost difference. For the scenario analyses the outcomes are the potential cost savings per 1000 patients when a decrease in iCSF leakage would be achieved.

Results Mean cost difference between patients with and without iCSF leakage was $€ 9665(95 \% \mathrm{Cl}$, €5125 to $€ 14$ 205). The main cost driver was hospital stay with a difference of 8.5 days. A $25 \%$ incidence reduction would result in a mean cost saving of -€94 $039(95 \% \mathrm{Cl}$, $-€ 218258$ to $-€ 7077)$ per 1000 patients. A maximum cost reduction of $-€ 653025(95 \% \mathrm{Cl},-€ 1204243$ to -€169 120) per 1000 patients could be achieved if iCSF leakage would be reduced with $75 \%$ in all patients, with 72 cases of iCSF leakage avoided.

Conclusions Postoperative iCSF leakage after intradural cranial surgery increases healthcare costs significantly and substantially. From a health economic perspective preventative measures to avoid iCSF leakage should be pursued.

\section{INTRODUCTION}

Cerebrospinal fluid (CSF) leakage is one of the most common complications after
Strengths and limitations of this study

- To our knowledge, this is the largest cost analysis providing a detailed breakdown of costs for incisional cerebrospinal fluid (iCSF) leakage after intradural cranial surgery.

- An advantage of the method applied in this study is the adaptability of the transparent model to other settings.

- One limitation of our approach is the effect of initial surgery costs on the results of our analyses.

- Although, this analysis contains the largest patient population in an economic evaluation of iCSF leakage, the number of patients in the individual categories for secondary complications and treatment modalities remains low.

neurosurgical intervention. The incidence of CSF leakage after intradural cranial surgery reported in the literature is $8 \%$ on average and depends on location of the surgery, indication of the surgery and patient-related risk factors. ${ }^{1}$ CSF leakage-related complications include wound infection and meningitis, and may necessitate prolonged hospital admission, external CSF drainage or reoperation. Therefore, CSF leakage is not only associated with substantial morbidity, but also with increased healthcare costs as well. ${ }^{1}$ Grotenhuis found that the total extra cost of CSF leakage is approximately $€ 12000$ for intradural cranial surgery, looking at the direct medical costs. ${ }^{2}$ Previous research, however, lacks specification of the main cost drivers and analysis of costs for specific treatment modalities for CSF leakage. Both the health and economic consequences of CSF leakage emphasise the importance of prevention of CSF leakage. 
Yet, preventative measures to reduce CSF leakage incidence may require financial input as well. Neurosurgeons closing themselves instead of residents, the use of devices, or increased operating room time because of a more precise closing technique to prevent CSF leakage may all lead to increased healthcare costs. Cost-benefit analyses of preventative strategies to reduce CSF leakage are lacking in the current body of literature.

In an increasingly cost aware healthcare system, financial implications of complications and their prevention are of great importance in deciding, which preventative strategies to pursue. Therefore, the health economic consequences should be considered as well when evaluating the efficacy of preventative strategies to avoid iCSF leakage.

The primary objective of the current study is to quantify the difference in healthcare consumption and associated costs between patients with CSF leakage after intradural cranial surgery and those without postoperative CSF leakage. The secondary objective is to quantify the economic effect per patient when a decrease in CSF leakage rate and related complications would be achieved using preventative measures that may require financial input.

\section{METHODS}

This cost analysis was performed from a hospital perspective, including detailed healthcare consumption of every individual patient. This study uses direct medical costs, without taking into account health insurance reimbursement.

Clinical data from a single centre were retrieved from previously collected retrospective international multicenter database (unpublished raw data). All consecutive adult patients undergoing intradural cranial surgery between 1 September 2017 and 1 September 2018 at the University Medical Center Utrecht were included. Patients who died within 1 month after surgery or were lost to follow-up were excluded, as for these patients there was insufficient certainty regarding the occurrence of the primary outcome measure (CSF leakage) introducing bias into the analysis and healthcare resources used during follow-up. Patients undergoing burr hole surgery or transsphenoidal surgery were excluded, as they represent separate patient categories with specific healthcare utilisation.

The following surgical characteristics had been collected: indication, urgency level, reoperation (yes/no), location of craniotomy (supratentorial or infratentorial), use of dural substitute and use of a dural sealant. Patient characteristics retrieved from the database included: age, sex, preoperative dexamethasone use, history of radiation therapy, diabetes, body mass index and smoking.

CSF leakage was defined as incisional cerebrospinal fluid (iCSF) leakage (either clinically diagnosed or confirmed through Beta-2 transferrin test) and did not include pseudomeningocele. Postoperative infection included superficial wound infection and deep wound infection and/or meningitis requiring treatment. The type of treatment was reviewed when iCSF leakage occurred. The treatment was divided into three categories: conservative treatment, external drainage placement and operative wound revision. Conservative treatment consisted of pressure bandage for wound compression and/or additional suture placement. First, a cost analysis was performed based on clinical and detailed cost data. This cost analysis was followed by scenario analyses to investigate the effect of reduction of iCSF leakage on health economic outcomes. A decision tree was used to combine the afore-mentioned cost analysis and the incidence rates of complications.

\section{Cost analysis}

Healthcare resources consumed by eligible patients from 30 days prior to 180 days after surgery were retrieved from medical records. Costs included readmissions and considers all-cause healthcare utilisation. Unit prices were retrieved from the Dutch Healthcare Authority (Nederlandse Zorgautoriteit), the cost manual of the National Healthcare Institute (Zorginstituut Nederland) and literature research and linked to the corresponding healthcare activities. ${ }^{34}$ The costs for an external ventricle drain and external lumbar drain and dural sealants were based on the existing literature and local prices. ${ }^{5}$ Costs for cranial surgery and reoperation were determined based on operating room time multiplied by cost per minute $(€ 10.59) .{ }^{6}$ All costs are presented in 2018 Euros.

Outcomes of the cost analysis included a detailed breakdown of mean costs per patient for patients with postoperative iCSF leakage and patients without. Different costs were divided into categories; outpatient visits, diagnostics, primary surgery, expensive drugs (eg, chemotherapy for patients with brain tumour), clinical admissions, other costs (eg, physiotherapy and dietetics), leakage treatment and sealant costs.

As well as the total healthcare costs for patients with CSF leakage stratified by treatment; reoperation, drain (external lumbar drain and external ventricle drain), reoperation and drain, and/or conservative treatment (including pressure bandage and additional sutures). Difference between groups was tested with Mann-Whitney $\mathrm{U}$ test since data were not normally distributed.

\section{Scenario analysis}

Model development

A decision tree was developed (online supplemental material 1) outlining intradural cranial surgery and the occurrence of complications, including iCSF leakage. This decision tree allows the quantification of the room for improvement in scenario analyses by adapting probabilities of individual events. This is achieved by multiplying the probability of a patient qualifying for a certain subgroup by the healthcare costs associated with these subgroups, online supplemental material 1 outlines the probabilities and subgroup costs used to recalculate 
healthcare costs. Outliers can impact outcomes significantly. To account for input parameter uncertainty distributions were fitted, beta distributions for probabilities and gamma distributions for costs. A probabilistic analysis with a Monte Carlo simulation with 10000 iterations was used to determine model outcomes and ranges.

\section{Scenario analyses}

Scenario analyses were performed to determine the health economic effects of reduction of iCSF leakage. Three different scenarios were applied to gain more information on the possible benefits of CSF reduction with various preventative strategies. (I) The iCSF leakage incidence use was decreased with $25 \%$ steps between $0 \%$ and $75 \%$. (II) The iCSF leakage incidence was reduced and weighted against varying costs of potential interventions of variable efficacy. (III) The first two scenario's applied for subgroups with different risk of iCSF leakage (supratentorial surgery and infratentorial surgery). Outcomes of the scenario analyses were presented as difference in costs and number of iCSF leakage cases avoided per 1000 patients was calculated as well as the number needed to treat $(\mathrm{NNT})$. To determine parameter influence on the outcome of the scenarios, a deterministic sensitivity analysis was performed and a tornado diagram was constructed.

\section{RESULTS}

In total, 616 consecutive patients were included in this study. Table 1 provides an overview of the patient characteristics. The mean age of patients was $53.5( \pm 15.8)$ years. The most common indication for surgery was tumour resection; 399 patients $(64.8 \%)$ and most patients had a supratentorial approach; 517 (83.9\%). A total of 59 patients had postoperative iCSF leakage (9.6\%).

\section{Cost per patient and detailed breakdown costs}

Average cost per patient and a detailed breakdown of costs are included for all of the 616 patients. In table 2, the average costs per patient with and without iCSF leakage are outlined. Five out of seven cost categories were higher for patients with iCSF leakage compared with patients without iCSF leakage. Costs for external ventricle drain, external lumbar drain and reoperation were categorised under treatment costs in table 2.

Difference in costs between patients without iCSF leakage and with iCSF leakage was $€ 9665$ (95\% CI, $€ 5125$ to $€ 14205$ ). Total average healthcare costs for patients without iCSF leakage was €20 498 (95\% CI, €19 183 to $€ 21$ 813) compared with €30 163 (95\% CI, €23 654 to $€ 36672$ ) for patients with iCSF leakage (table 2). When comparing costs incurred starting from the day of primary surgery (days $0-180$ ), costs were $€ 17759$ (95\% CI, $€ 16497$ to $€ 19021$ ) for patients without iCSF leakage and $€ 28105$ (95\% CI, €21 695 to $€ 34515$ ) for patients with iCSF leakage.

The main reason for the difference in cost, over both the total time and the postoperative time, was the significant difference in length of hospital stay, for which costs are categorised as clinical admissions. Difference in length of stay (LOS) was 8.5 days (95\% CI, 5.3 to 11.7). For patients without incisional leakage, LOS was 12.8 days (95\% CI, 11.9 to 13.8 ) and for patients with iCSF leakage LOS was 21.3 days (95\% CI, 16.6 to 26.1). Furthermore, the incidence of secondary complications was significantly higher in the iCSF group. Highest costs among subgroups were found for patients with deep wound infection and/ or meningitis ( $€ 39323$ to $€ 57$ 862). Patients without additional complications had the lowest costs among all subgroups (€19 050 to $€ 26797$ ) (table 3 ).

For supratentorial surgery, there was a significant cost difference between patients with iCSF leakage ( $€ 20180$,

Table 1 Patient characteristics

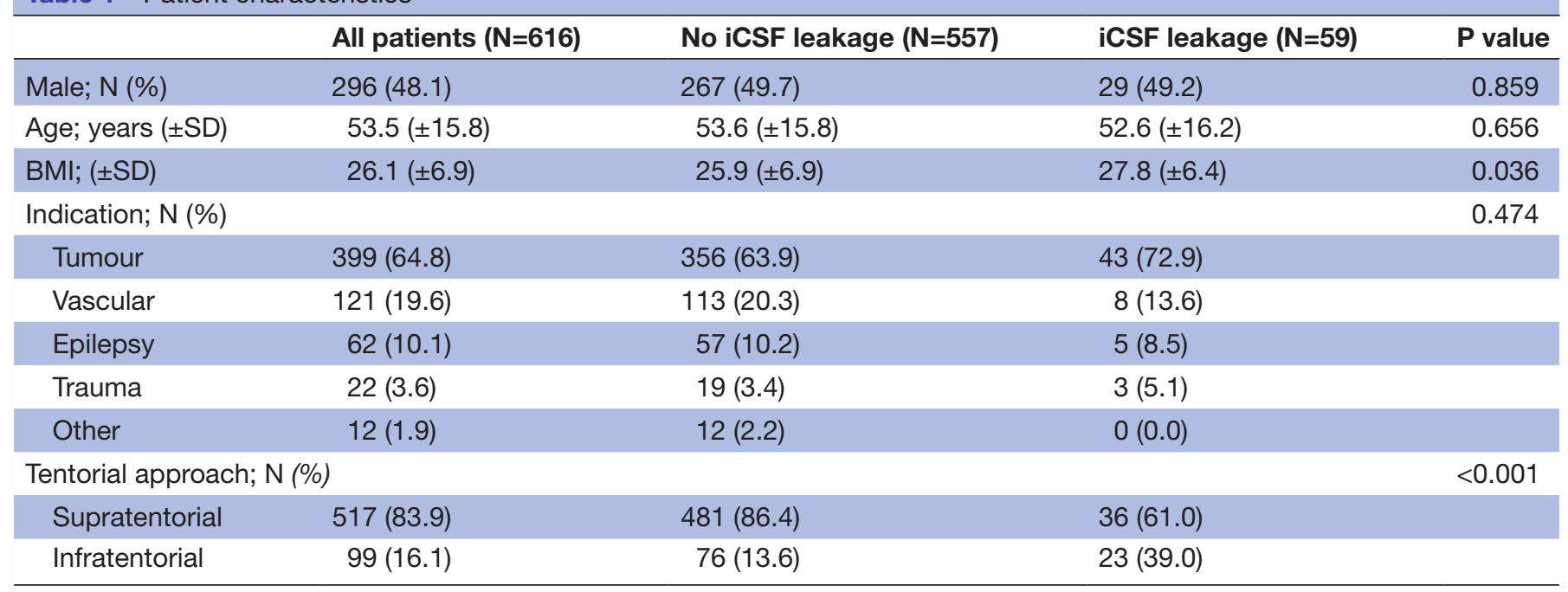

$P$ values smaller than 0.05 are considered significant.

BMI, body mass index; iCSF, incisional cerebrospinal fluid. 


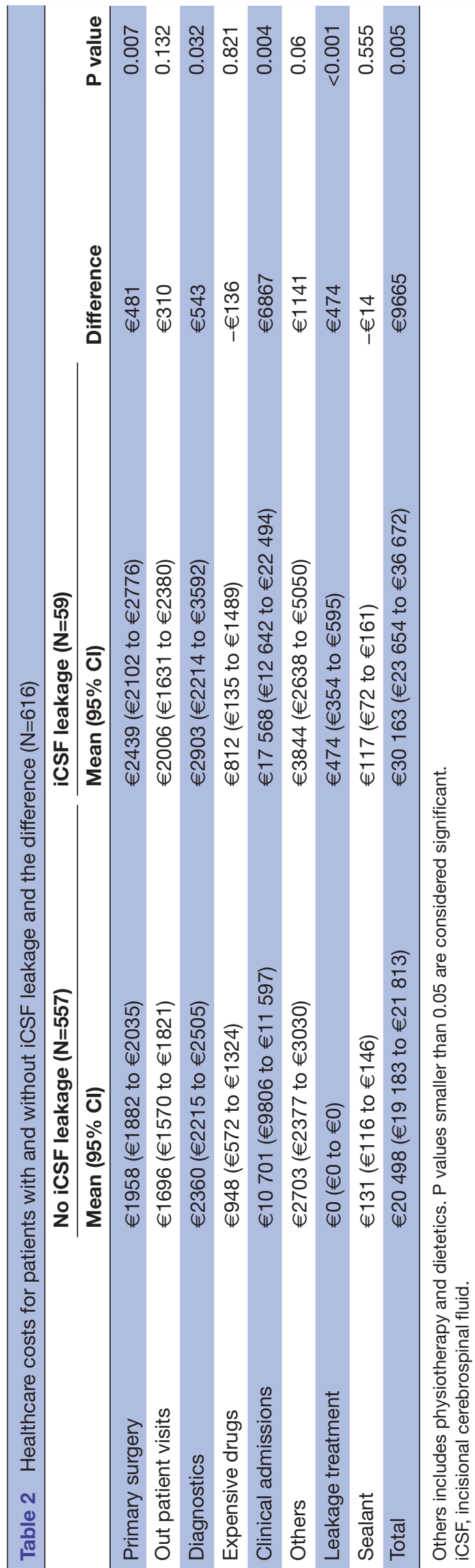

$\pm € 14$ 504) and those without (€31 $219 \pm € 25$ 224). For infratentorial surgery, patients with iCSF leakage had a mean cost of $€ 28510$ ( $\pm € 25057)$ as compared with $€ 22.512$ ( $\pm € 22369$ ) for patients without iCSF leakage. This difference was not statistically significant (table 3 ).

In the group of patients with postoperative iCSF leakage $(\mathrm{N}=59), 18$ patients received conservative treatment, 7 patients required reoperation, 26 patients were treated with an external CSF drain and 8 patients required reoperation and a drain. In the group of patients treated conservatively, 10/18 had CSF leakage once or two times. All other patients with CSF leakage had continuous leakage. Table 4 shows the total healthcare costs and LOS for patients with iCSF leakage stratified per treatment modality. Lowest costs were found for the 18 patients who were treated conservatively (€21 046 ( $₫ € 11433)$ ). Highest costs were found for the seven patients requiring reoperation; $€ 36117$ ( $\pm € 45$ 056). Longest LOS was for patients requiring reoperation and drain; 26.5 days $( \pm 17.6$ days $)$. There was no statistically significant difference in LOS or costs between patients who were treated conservatively and those who underwent reoperation. Patients who were treated with external CSF drainage or reoperation and external CSF drainage combined had significantly longer LOS and higher costs compared with patients who were treated conservatively. No significant differences in LOS or costs were found between invasive treatment modalities.

\section{Scenario analyses}

Table 5 presents an overview of the outcomes of scenario analysis I and III.

Figure 1 shows the potential cost savings per patient when a decrease in iCSF leakage would be achieved. A maximum cost reduction of $-€ 653025$ (95\% CI, $-€ 1204$ 243 to $-€ 169120)$ per 1000 patients could be achieved if iCSF leakage would be reduced with $75 \%$. The number of cases avoided would be 72. The NNT in this scenario is 14. For supratentorial surgery reduction of iCSF leakage with $25 \%-75 \%$ would lead to significant cost reduction and a maximum of 53 cases of iCSF leakage avoided. For infratentorial surgery there is a trend towards substantial cost savings for reduction rates between 25\% and 75\%; however, this is not significant.

If costs of potential preventative strategies are added to accomplish iCSF leakage (scenario II and III) our model shows cost reduction for measures at a price of $€ 250$ per patient at an iCSF leakage reduction of $50 \%-75 \%$ in all patients and both subgroups. Preventative strategies at a price of $€ 500$ euro per patient only lead to cost savings in all patients and supratentorial cases if they reduce iCSF leakage with $75 \%$. For infratentorial cases, this scenario results in cost savings at a 50\% reduction as well. Preventative strategies that cost $€ 750$ per patient lead to cost reduction only when applied in infratentorial cases with an iCSF leakage reduction of $75 \%$ (figure 2 ).

The deterministic sensitivity analysis showed that the parameter with the greatest influence on scenario 
Table 3 Average total healthcare costs per patient for different subgroups based on approach and complication

\begin{tabular}{|c|c|c|c|c|c|c|c|}
\hline & \multicolumn{3}{|c|}{ No iCSF leakage $(\mathrm{N}=557)$} & \multicolumn{3}{|c|}{ iCSF leakage $(\mathrm{N}=59)$} & \multirow[b]{2}{*}{ P value } \\
\hline & $\mathbf{N}$ & Mean & SD & $\mathbf{N}$ & Mean & SD & \\
\hline Supratentorial & 481 & $€ 20180$ & $€ 14504$ & 36 & $€ 31219$ & $€ 25224$ & 0.014 \\
\hline Superficial wound infection & 10 & $€ 31616$ & $€ 32380$ & 10 & $€ 30448$ & $€ 22020$ & 0.926 \\
\hline Deep wound infection and/or meningitis & 14 & $€ 48881$ & $€ 24918$ & 8 & $€ 42130$ & $€ 37982$ & 0.557 \\
\hline No complications & 73 & $€ 21883$ & $€ 22383$ & 16 & $€ 25163$ & $€ 16561$ & 0.574 \\
\hline Superficial wound infection & 2 & $€ 27804$ & $€ 11027$ & 2 & $€ 28248$ & $€ 16988$ & 0.978 \\
\hline Deep wound infection and/or meningitis & 1 & $€ 57862$ & - & 5 & $€ 39323$ & $€ 50566$ & 0.755 \\
\hline
\end{tabular}

$P$ values smaller than 0.05 are considered significant.

iCSF, incisional cerebrospinal fluid.

outcomes was costs for patients without iCSF leakage and an infratentorial approach. Lowest influence was found for the incidence of iCSF leakage in infratentorial patients (figure 3).

\section{DISCUSSION}

There is a substantial and significant cost difference of $€ 9665$ between patients with postoperative iCSF leakage after intradural cranial surgery and those without. The average healthcare cost for cranial intradural surgery ranges between $€ 20498$ for patient without iCSF leakage and $€ 36117$ for patients with reoperation, which was the most expensive. A maximum cost reduction of $-€ 653025$ (95\% CI, -€1 204243 to $-€ 169$ 120) per 1000 patients could be achieved if iCSF leakage would be reduced with $75 \%$ in all patients.

Our model shows that reducing leakage rates could lead to substantial cost reduction, even if financial input is required. However, whether the use of preventative measures that require financial input in all patients or a subgroup of patients at risk results in cost savings depends on their price and efficacy. Because of the higher risk of iCSF leakage in infratentorial surgery, more expensive preventative measure of a certain efficacy could still lead to cost savings in this subgroup, when they do not for the total population.

To our knowledge, this is the largest cost analysis providing a detailed breakdown of costs for iCSF leakage after intradural cranial surgery. Furthermore, it is the first study applying a model to calculate the health economic effects of improved preventative measures. An advantage of the method applied in this study is the adaptability of the transparent model to other settings. If other hospitals are aware of their leakage rate and healthcare costs, this method could be used to estimate possible future cost savings, for example with improved sealants.

One limitation of our approach is the effect of initial surgery costs on the results of our analyses. Despite this being the most comprehensive method of taking into account all associated costs, it may be the case that part of the cost difference is driven by the initial surgery, as complex and longer surgeries are more expensive. Second, we have collected healthcare consumption in a single centre. There is thus a theoretical risk of missing the costs of patients that may have received follow-up treatment elsewhere, without this being communicated to the primary centre. As patients with loss to follow-up were excluded from the initial database and treatment of complications in a different centre is unusual, we do not believe this has affected the outcomes of the current study.

Thereby, although this analysis contains the largest patient population in an economic evaluation of iCSF leakage, the number of patients in the individual categories for secondary complications and treatment modalities remains low. It is, therefore, difficult to interpret cost differences for specific secondary complications in detail. In these limited numbers of cases, heterogeneity of patients could be the main difference between those with iCSF leakage and those without. Results of the comparisons between the different treatment modalities should be interpreted with some caution as well, for the same reason. Especially, the subgroup of patients who underwent reoperation is limited in size and has large SD of both the LOS and the costs. Furthermore, these limited subgroups led to larger uncertainty around the scenario analyses modelling the potential health economic effects of iCSF leakage reduction, especially for the infratentorial subgroup. Another limitation of the scenario analyses is the linear reduction in iCSF leakage, which assumes that iCSF leakage can be prevented with a certain efficacy across the total population. It may, however, be the case that for certain subgroups iCSF leakage cannot be avoided with preventative measures.

These results are based on healthcare consumption and costs of one centre in the Netherlands. Therefore, applying these results to different countries is challenging. Differences in clinical practice and prices, for 

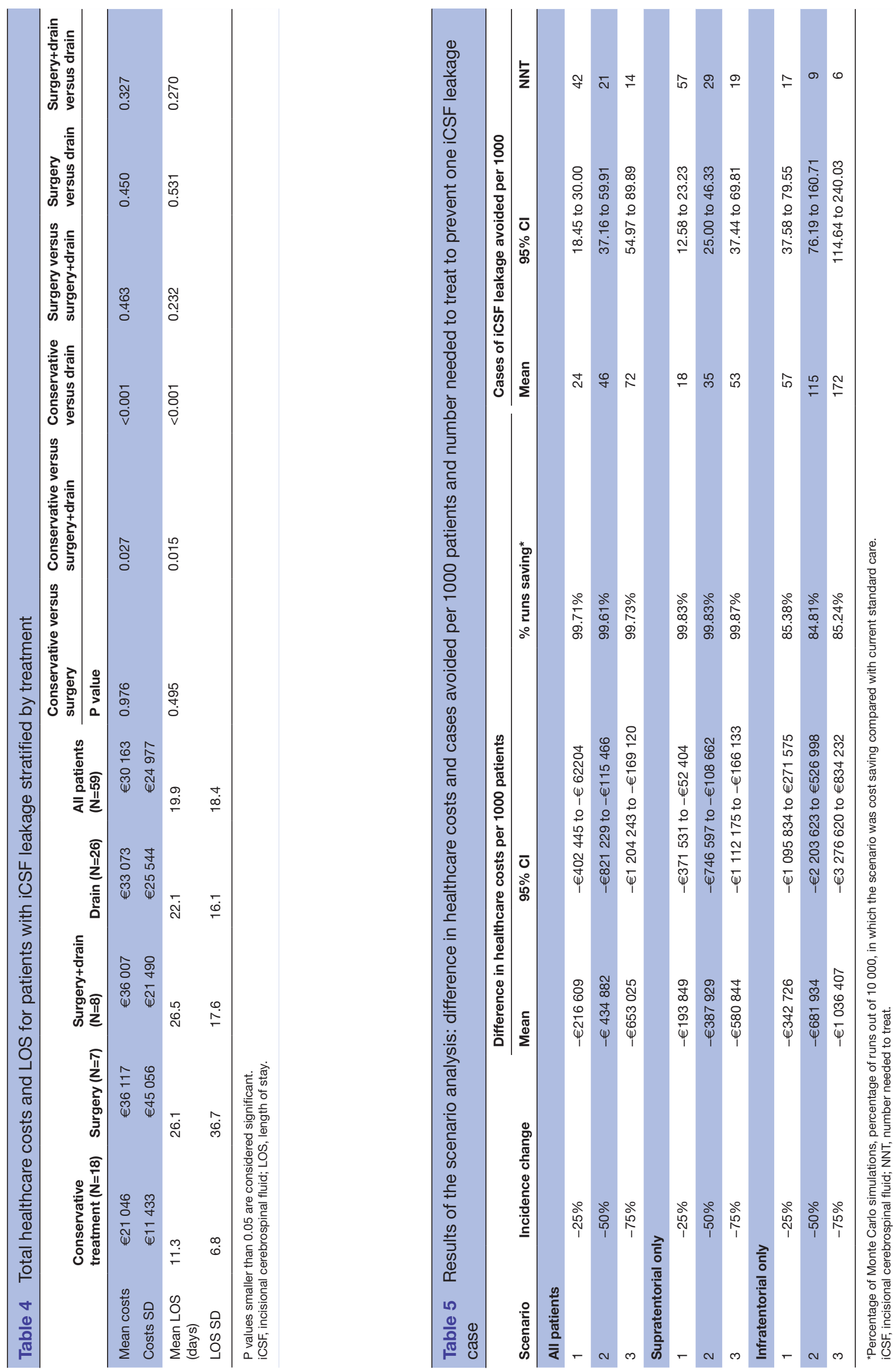


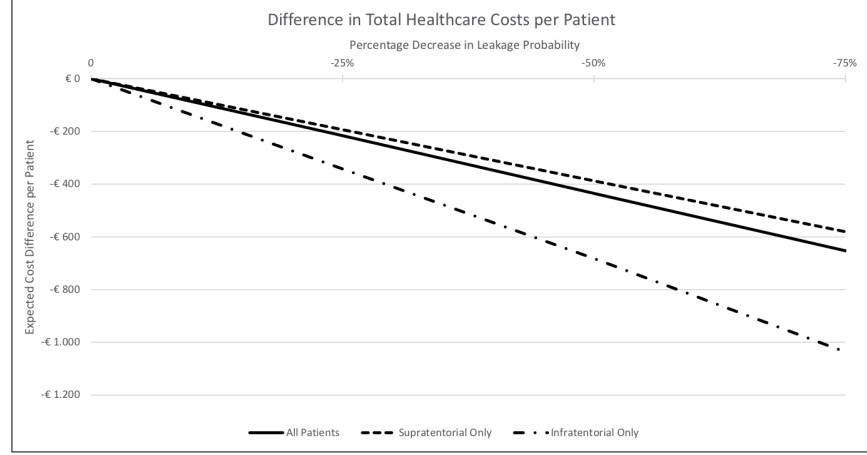

Figure 1 Potential costs savings per patient with a decrease in incisional cerebrospinal fluid leakage.

instance, may influence the effects observed in this study considerably. ${ }^{7}$ It is thus recommended that data on cost prices and resource use should be obtained from or adapted to the setting of interest. ${ }^{7}$ Furthermore, baseline risk should be location specific, whereas treatment effect may be more generalisable. ${ }^{7}$ Although, larger differences are to be expected between the healthcare systems across continents, even within western Europe economic analyses of medicines vary significantly. ${ }^{7}$

The additional healthcare costs for patients with incisional CSF leakage in this study are comparable to those found by Grotenhuis in the Netherlands, who found a cost difference of approximately $€ 12000$, for cranial surgery including transsphenoidal procedures. ${ }^{2}$ Our study includes all healthcare resources consumed within a predefined time frame, whereas Grotenhuis based calculations on certain cost categories only. Another study from Germany by Piek et al calculated cost differences between patients with and without CSF leakage in detail and found a comparable result of $€ 11.420$. $^{8}$ Their study, however, also included subcutaneous CSF collections as CSF leaks, and it has a limited sample size of 168 patients (of which only three had percutaneous CSF leaks). ${ }^{8}$

The breakdown of costs shows that clinical admission is the main cost driver for the difference between patients with and without iCSF leakage. Patients with iCSF leakage have higher risk of infection or meningitis. ${ }^{9}$ These complications may further explain the cost difference between patients with and without iCSF leakage as they require prolonged clinical admission. These results are in line with the study of Parikh et al that identified

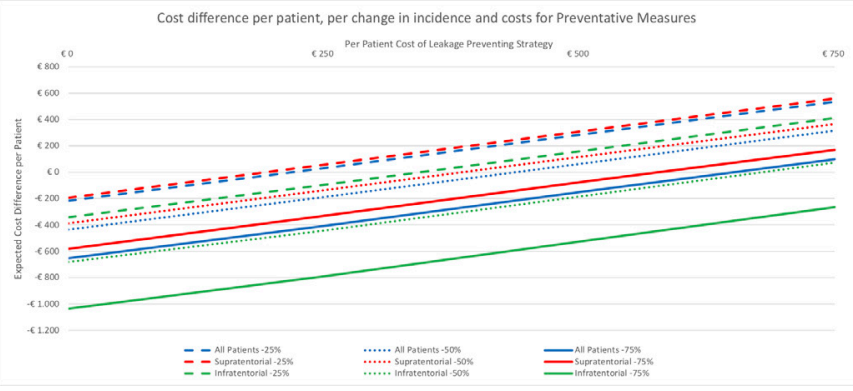

Figure 2 Cost difference per patient, per change in incidence and cost for preventative measure.
Tornado Diagram Deterministic Sensitivity Analysis

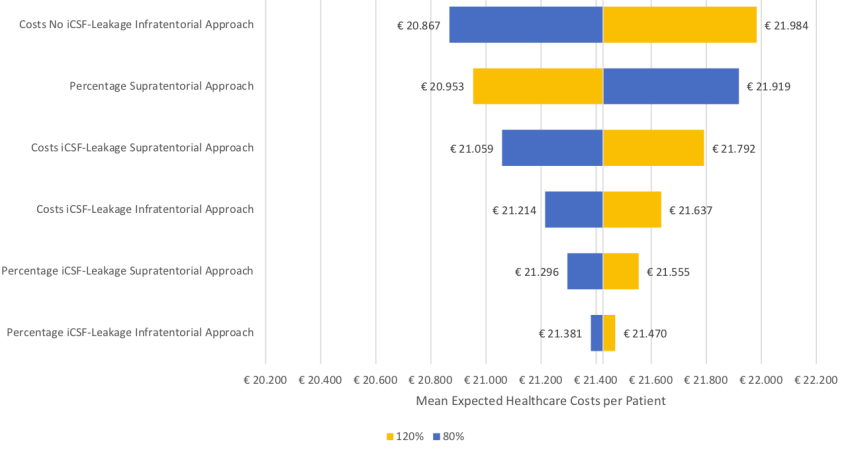

Figure 3 Tornado diagram indicting the influence of the different input parameters of the model.

increased LOS and the association of CSF leakage with secondary complications such as meningitis as the main reasons for increased healthcare costs after transsphenoidal surgery. ${ }^{10}$

Additionally, the costs for interventional treatment of iCSF leakage are a substantial cost driver, considering that patients who can be managed conservatively have total average costs that are comparable to patients without iCSF leakage. In the group of patients managed conservatively, though, 10/18 patients $(55.6 \%)$ did not have continuous iCSF leakage, but incisional leakage that occurred once or twice, suggestive of a subcutaneous pocket that has discharged. All patients that had to be managed with invasive treatment had continuous iCSF leakage. Patients treated with an external CSF drain have significantly longer LOS and higher costs compared with those treated conservatively. Contrary to Parikh et $a l$ we did not find shorter LOS in patients treated with reoperation compared with those treated with external CSF drainage only. ${ }^{10}$ This may imply that reoperation as a treatment for iCSF leakage is performed sooner after endoscopic endonasal surgery than after craniotomy. An advantage of reoperation compared with external CSF drainage is the quick return to mobilisation as opposed to bedrest required during external CSF drainage. This is not reflected in a difference in LOS between these patients in our population, however. Besides a delay in surgical treatment, other factors related to recovery such as comorbidity may explain why LOS is similar for these treatment modalities.

This study confirms that from a health economic perspective, iCSF leakage should be reduced. Improved preventative strategies reducing the iCSF leakage rate, even though they may add to the overall healthcare costs per patient, could be beneficial from an economic standpoint. Furthermore, increased understanding of risk factors for iCSF leakage and associated costs may contribute to improving the indication for use of currently available and future methods of augmented dural closure. Considering that conservative treatment for continuous iCSF leakage is rarely effective, early interventional treatment for this group is recommended. 
Furthermore, methods that shorten LOS for patients with external CSF drains should be investigated. Our model of the health economic effects of iCSF leakage and potential cost savings of improved preventative strategies should be applied to different healthcare settings to evaluate the cost difference and potential cost savings location specifically to assist physicians and healthcare managers in decision-making regarding preventative strategies to avoid iCSF leakage in their situation.

\section{Author affiliations}

${ }^{1}$ Julius Center for Health Sciences and Primary Care, University Medical Center Utrecht, Utrecht, The Netherlands

${ }^{2}$ Neurology and Neurosurgery, University Medical Centre Utrecht Brain Centre, Utrecht, The Netherlands

${ }^{3}$ Translational Neuroscience, University Medical Centre Utrecht Brain Centre, Utrecht, The Netherlands

${ }^{4}$ Neuro-oncology, Princess Maxima Center for Pediatric Oncology, Utrecht, The Netherlands

${ }^{5}$ Neurosurgery, Clinical Neuroscience Center, University Hospital Zurich, Zurich, Switzerland

Contributors Conceptualisation: TPCvD and GWJF. Methodology: GWJF, CvL, EMHS and TPCVD. Formal analysis and investigation: CvL and EMHS. Writing - original draft preparation: CVL and EMHS. Writing —review and editing: TPCVD, GWJF, EWH, AK, MK, CVL and EMHS. Resources: AK and MK. Supervision: TPCVD, GWJF and EWH. Guarantor: TPCVD and GWJF.

Funding EMHS and AK received a research grant through Polyganics B.V., grant number N/A.

Competing interests CVL and GWJF received a consultancy fee through Polyganics B.V. for this research.

Patient consent for publication Not applicable.

Ethics approval This study does not involve human participants.

Provenance and peer review Not commissioned; externally peer reviewed.

Data availability statement The data set that was analysed for this study is available from the corresponding author upon reasonable request.

Supplemental material This content has been supplied by the author(s). It has not been vetted by BMJ Publishing Group Limited (BMJ) and may not have been peer-reviewed. Any opinions or recommendations discussed are solely those of the author(s) and are not endorsed by BMJ. BMJ disclaims all liability and responsibility arising from any reliance placed on the content. Where the content includes any translated material, BMJ does not warrant the accuracy and reliability of the translations (including but not limited to local regulations, clinical guidelines, terminology, drug names and drug dosages), and is not responsible for any error and/or omissions arising from translation and adaptation or otherwise.

Open access This is an open access article distributed in accordance with the Creative Commons Attribution Non Commercial (CC BY-NC 4.0) license, which permits others to distribute, remix, adapt, build upon this work non-commercially, and license their derivative works on different terms, provided the original work is properly cited, appropriate credit is given, any changes made indicated, and the use is non-commercial. See: http://creativecommons.org/licenses/by-nc/4.0/.

\section{ORCID iD}

Emma M H Slot http://orcid.org/0000-0002-4296-1331

\section{REFERENCES}

1 Kinaci A, Algra A, Heuts S, et al. Effectiveness of dural sealants in prevention of cerebrospinal fluid leakage after craniotomy: a systematic review. World Neurosurg 2018;118:368-76.

2 Grotenhuis JA. Costs of postoperative cerebrospinal fluid leakage: 1-year, retrospective analysis of 412 consecutive nontrauma cases. Surg Neurol 2005;64:490-3.

3 Hakkaart-van Rooijen L, Van der Linden N, Bouwmans CAM, et al. Manual for cost analyses, methods and standard prices for economic evaluations in healthcare. Diemen, The Netherlands: Zorginstituut Nederland, 2015

4 Nederlandse Zorgauthoriteit. Performance and Tariffs specialist medical care, 2017. Available: https://puc.overheid.nl/doc/PUC_ 21709_22/1 [Accessed 3 Feb 2020].

5 Ferko $\bar{N}$, Corral M, Hogan A, et al. A hospital cost analysis of a fibrin sealant patch in soft tissue and hepatic surgical bleeding. ClinicoEconomics and Outcomes Research 2019;8:507-19.

6 Kluytmans A, Tummers M, van der Wilt GJ, et al. Early assessment of Proof-of-Problem to guide health innovation. Value Health 2019;22:601-6.

7 Drummond M, Barbieri M, Cook J, et al. Transferability of economic evaluations across jurisdictions: ISPOR good research practices Task force report. Value Health 2009;12:409-18.

8 Piek J, Weber C, Kundt G, et al. Pharmacoeconomical consequences of postoperative CSF leaks after intracranial surgery--a prospective analysis. J Neurol Surg A Cent Eur Neurosurg 2012;73:25-8.

9 Kourbeti IS, Vakis AF, Ziakas P, et al. Infections in patients undergoing craniotomy: risk factors associated with post-craniotomy meningitis. J Neurosurg 2015;122:1113-9.

10 Parikh A, Adapa A, Sullivan SE, et al. Predictive factors, 30-day clinical outcomes, and costs associated with cerebrospinal fluid leak in pituitary adenoma resection. J Neurol Surg B Skull Base 2020;81:43-55. 